\title{
Hemodynamic Responses of Rat Brain Measured by Near-infrared Spectroscopy During Various Whisker Stimulations
}

\author{
Seungduk Lee, Dalkwon Koh, and Kiwoon Kwon \\ Department of Biomedical Engineering, Yonsei University, Wonju 220-842, Korea \\ Hyun Joo Lee, Yiran Lang, and Hyung-Cheul Shin \\ Department of Physiology, Hallym University, Chuncheon 220-702, Korea \\ Beop-Min Kim* \\ Department of Biomedical Engineering, Korea University, Seoul 136-703, Korea
}

(Received August 25, 2008 : revised October 10, 2008 : accepted December 19, 2008)

\begin{abstract}
NIRS (Near-infrared spectroscopy) is a relatively, new, non-invasive, and non-ionizing method of measuring hemodynamic responses in thick biological tissues such as the cerebral cortex. In this study, we measured the hemodynamic responses of the rat barrel cortex to whisker stimulation by using a frequency-domain NIRS system. We designed multiple optical probes comprising multimode optical fibers and manipulating arms, both of which can be easily applied to small animals. Various electrical stimulations were applied to rat whiskers at different voltage levels and stimulation frequencies. Our results show that the hemodynamic responses are highly dependent on the stimulation voltage level, and not so much on stimulation frequency. This paper suggests that NIRS technology is highly suitable for the study of small animal brains.
\end{abstract}

Keywords: Near-infrared spectroscopy (NIRS), Hemodynamic responses, Neuroimaging, Neurovascular coupling, Whisker stimulation

OCIS codes : (170.3880) Medical and biological imaging; (170.1470) Blood/tissue constituent monitoring; (170.3660) Light propagation in tissue

\section{INTRODUCTION}

In neuroscience, small animals such as rats and knock-in and knock-out mice can be used as models of neurological disease, including stroke, epilepsy, and Alzheimer's disease. Various neuroimaging technologies are employed to approach this problem including positron emission tomography (PET), functional magnetic resonance imaging (fMRI) and near-infrared spectroscopy (NIRS) [1].

PET images glucose metabolism using radioisotopes such as 2-deoxy-2 $\left[{ }^{18} \mathrm{~F}\right]$ fluoro-d-glucose $\left({ }^{18} \mathrm{FDG}\right)$ in the brain. However, these isotopes can be harmful to the human body. It is known that this device is very expen-

*Corresponding author: bmk515@korea.ac.kr sive and has the drawback of requiring too much time to reconstruct images [2]. fMRI now is used as a universal imaging tool for neuroscience but it also has some disadvantages such as low signal-to-noise ratio of bloodoxygen level dependence (BOLD) signal level, low temporal resolution and high cost. It is especially problematic to use PET or fMRI in small animal studies due to the small brain sizes of small animals [3]. Even if our study is focused on hemodynamic signal detection and not on imaging, NIRS can be used for imaging purposes if a larger number of optical probes are used. Although NIRS has some disadvantages such as low spatial resolution and low penetration depth, it is an emerging tool for neuroscience since it costs less to build, shows high temporal resolution and uses harmless light. 
NIRS is used to measure concentrations of oxy- $\left(\mathrm{HbO}_{2}\right)$ and deoxy-hemoglobin (Hbr) using absorption spectra variations in the near-infrared band. The first attempt to measure oxygenation in biological tissues was done by Franz Jobsis, in 1977 [4]. Since then, NIRS-based research has focused on various biological areas including neuroscience [5-8]. In recent years, interest has turned to using NIRS to study neurovascular coupling, which occurs between neuronal activities and hemodynamic responses when neurons are activated. Although many research groups have investigated these correlation phenomenon, it has not been clearly elucidated [8]. Depending on experiment conditions and stimulus parameters, neurovascular coupling has been found to be linear [9-10] or nonlinear [11-13]. In order to understand these linear or nonlinear phenomena, multiple imaging (or detection) modalities have been combined. For example, measurements using magnetoencephalogram (MEG) or electroencephalogram (EEG) have been studied simultaneously [14].

In this study, we measured electrical/hemodynamic responses of rat brain using a home-made probe to record EEG and NIRS signals simultaneously while stimulating whisker areas. Various stimulus parameters were used and corresponding hemodynamic responses including peak values of $\mathrm{HbO}_{2}$ concentration were recorded.

\section{MATERIALS AND METHODS}

\section{II-1. Experimental setup}

All animal experiments followed the experimental prototype of the Department of Physiology at Hallym University. We used three adult female Sprague-Dawley rats with body weight $300 \sim 350 \mathrm{~g}$ each. Each rat was anesthetized with urethane $(1.25 \mathrm{~g} / \mathrm{kg})$ and the rat head was fixed to a stereotaxic frame to eliminate motion artifact. A heating pad (HB 101/2; LSI Letica) kept the rat's body temperature at a constant $37^{\circ} \mathrm{C}$. After the scalp of each rat was resected, we placed the optical probe to the right barrel cortex which would be activated when the left whisker was stimulated (Fig. 1 (a)). Two optical fibers with 400 um core diameter carrying different wavelengths were carefully tied-up and co-localized on one point with the help of an optical manipulating arm (Fig. 1 (b)). Additionally, to leave no air gap between the fiber tip and skull, thus simplifying the boundary condition, dental cement was applied at the boundary.

The bio-stimulator electrodes (Stimulator 100, Palmer Bioscience) were inserted at B4 and E1 sites of the left whisker [15]. The location of the cerebral barrel cortex, which is known to respond to the whisker stimulation, extends $2 \mathrm{~mm}$ in posterior direction from the bregma point and $5 \mathrm{~mm}$ in lateral direction from the medial point.

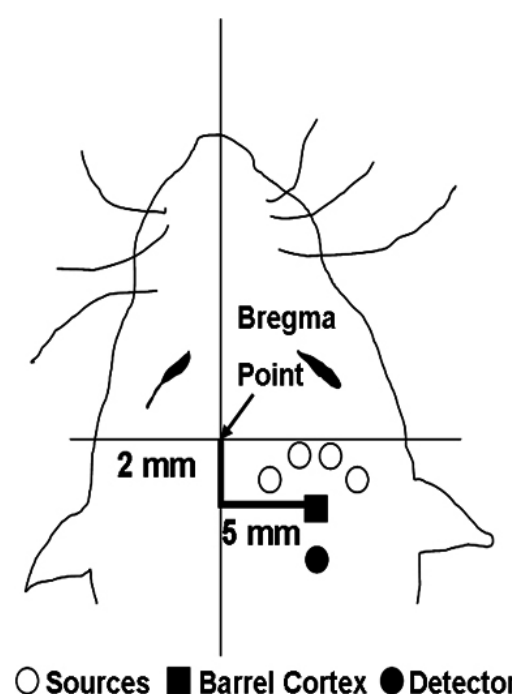

(a)

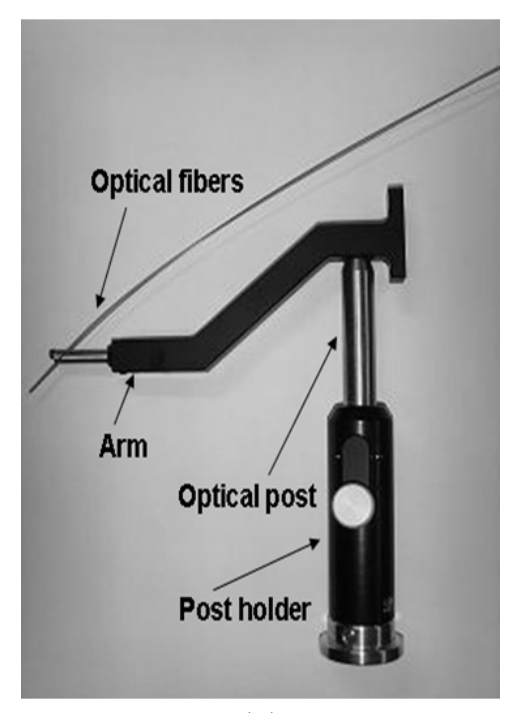

(b)

FIG. 1. (a) Arrangement of optical probes on the rat brain and (b) picture of the optical probe

We used the 8-channel frequency domain NIRS system (Imagent ${ }^{\text {TM }}$, ISS Corp.) which is composed of eight laser diode pairs (690 and $830 \mathrm{~nm})$ modulated at 110 $\mathrm{MHz}$ as sources and two photomultiplier tubes as a detector, which gain is modulated at $110.005 \mathrm{MHz}$. We used heterodyne detection schematic and detected signal modulated at $5 \mathrm{kHz}$. The pulse width of the electrical stimulation was fixed at $20 \mathrm{msec}$ and the total stimulation period was 6 sec. We varied both the voltages (i.e., 2, 5, and $10 \mathrm{~V}$ ) and frequencies (i.e., 1, 3, and $5 \mathrm{~Hz}$ ) in this study. Before stimulation, data recorded for $5 \mathrm{~min}$ were used as a baseline. After each set of $6 \mathrm{sec}$ stimulation, we allowed $3 \mathrm{~min}$ for recovery and repeated each procedure in triplicate (Fig. 2). The data-acquisition frequency was $20 \mathrm{~Hz}$ for all cases. 


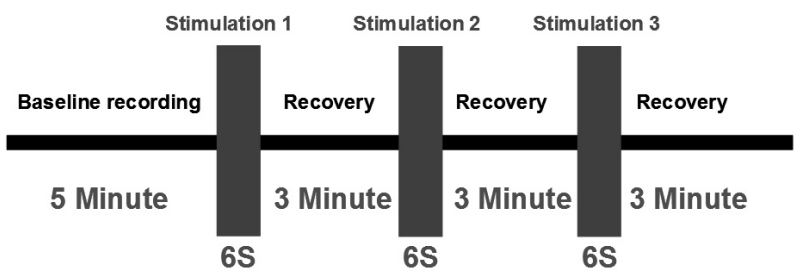

FIG. 2. Stimulation protocol

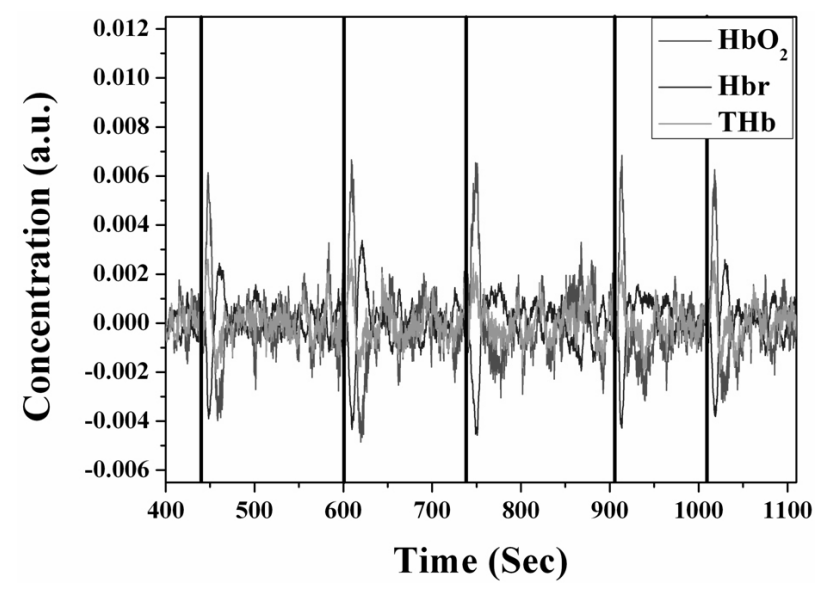

FIG. 3. Relative changes in $\mathrm{HbO}_{2}, \mathrm{Hbr}$, and $\mathrm{THb}$ by induced whisker stimulation (red: $\mathrm{HbO}_{2}$, blue: $\mathrm{Hbr}$, green: $\mathrm{THb}$, black: stimulation on)

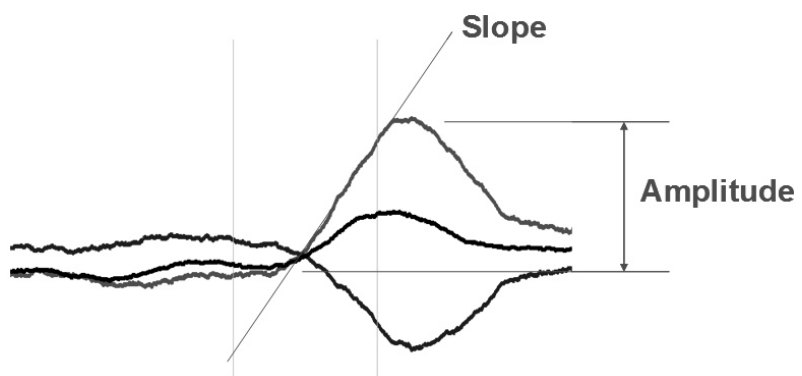

FIG. 4. Slope and peak value of $\mathrm{HbO}_{2}$ concentration

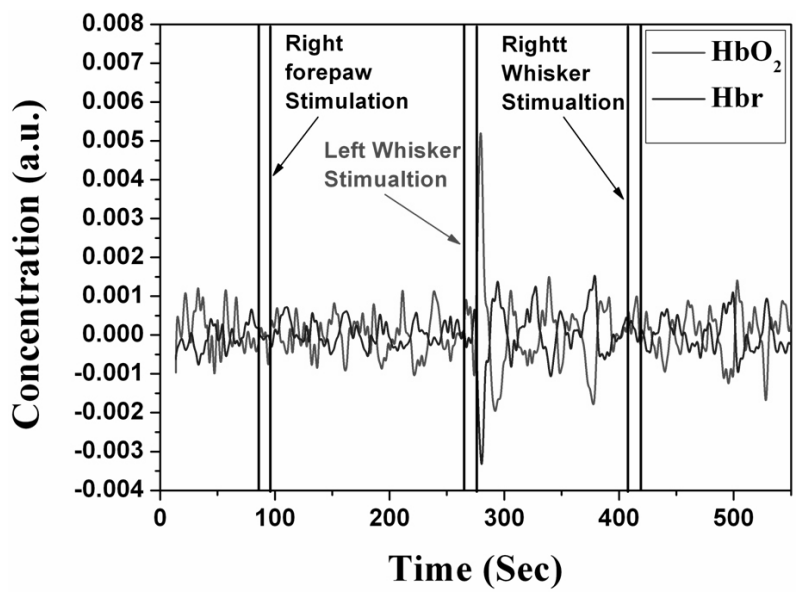

FIG. 5. Hemodynamic responses at various somatosensory cortices

II-2. Calculation of hemoglobin concentration
We calculated the concentrations of $\mathrm{HbO}_{2}$ and $\mathrm{Hbr}$ using the modified Beer-Lambert law [16] which requires intensity information in two different wavelengths:

$$
\Delta O D^{\lambda}=-\ln \frac{I_{\text {Final }}}{I_{\text {Initial }}}=\left(\epsilon_{\mathrm{HbO}_{2}}^{\lambda} \Delta\left[\mathrm{HbO}_{2}\right]+\epsilon_{\mathrm{HbO}}^{\lambda} \Delta[\mathrm{Hbr}] B^{\lambda} L\right.
$$

Where $I_{\text {initial }}$ is the source light intensity, $I_{\text {final }}$ is the light intensity at the detector, $\epsilon$ is the absorption coefficient, $\mathrm{B}$ is the differential pathlength factor, $\mathrm{L}$ is the source-detector distance, $\mathrm{HbO}_{2}$ is the oxy-hemoglobin, $\mathrm{Hbr}$ is the deoxy-hemoglobin and $\lambda_{1}$ and $\lambda_{2}$ are 690 and $830 \mathrm{~nm}$, respectively.

$$
\Delta[\mathrm{Hbr}]=\frac{\epsilon_{\mathrm{Hb} \mathrm{O}_{2}}^{\lambda_{2}} \frac{\Delta O D^{\lambda_{1}}}{B^{\lambda_{1}}}-\epsilon_{\mathrm{HbO} O_{2}}^{\lambda_{1}} \frac{\Delta O D^{\lambda_{2}}}{B^{\lambda_{2}}}}{\left(\epsilon_{\mathrm{Hbr}}^{\lambda_{1}} \epsilon_{\mathrm{HbO} O_{2}}^{\lambda_{2}}-\epsilon_{\mathrm{Hbr}}^{\lambda_{2}} \epsilon_{\mathrm{HbO} O_{2}}^{\lambda_{1}}\right) L}
$$

and

$$
\Delta\left[H b O_{2}\right]=\frac{\epsilon_{H b r}^{\lambda_{1}} \frac{\Delta O D^{\lambda_{2}}}{B^{\lambda_{2}}}-\epsilon_{H b r}^{\lambda_{2}} \frac{\Delta O D^{\lambda_{1}}}{B^{\lambda_{1}}}}{\left(\epsilon_{H b r}^{\lambda_{1}} \epsilon_{H b O_{2}}^{\lambda_{2}}-\epsilon_{H b r}^{\lambda_{2}} \epsilon_{H b O_{2}}^{\lambda_{1}}\right) L}
$$

The total hemoglobin $(\mathrm{THb})$ is the sum of $\mathrm{HbO}_{2}$ and Hbr. Data collected were analyzed and the relationship between the stimulation parameters and the corresponding hemodynamic responses was studied.

\section{RESULTS}

Fig. 3 illustrates the data collected while stimulating the whole left-whisker area using a piece of stick. Whisker stimulation lasted for about $10 \mathrm{sec}$ and was repeated five times. Between each stimulation, $3 \mathrm{~min}$ of recovery time was allowed. Approximately within 1 1.5 sec after stimulation, the $\mathrm{HbO}_{2}$ concentration started to increase. Also, we noticed that the concentration reached a peak value within $6 \sim 8 \mathrm{sec}$ of stimulation. On the other hand, the peak value of $\mathrm{Hbr}$ concentration decreased in similar time scale. However, compared to the $\mathrm{HbO}_{2}$ values, the peak value of Hbr change was approximately $60 \%$.

Fig. 4 is part of the signal in Fig. 3 which shows typical hemodynamic responses to whisker stimulation. We extracted two different parameters from these data: peak and slope values of $\mathrm{HbO}_{2}$ changes. A linear-fitting was conducted to calculate the slope value and all peak and slope values for $\mathrm{HbO}_{2}$ concentration were averaged over the whole stimulation sequence.

To verify that our signal originated only from the left-side whisker, we stimulated the forepaw and the right side of the whisker. Fig. 5 shows that no significant hemodynamic responses are observed by stimulation 


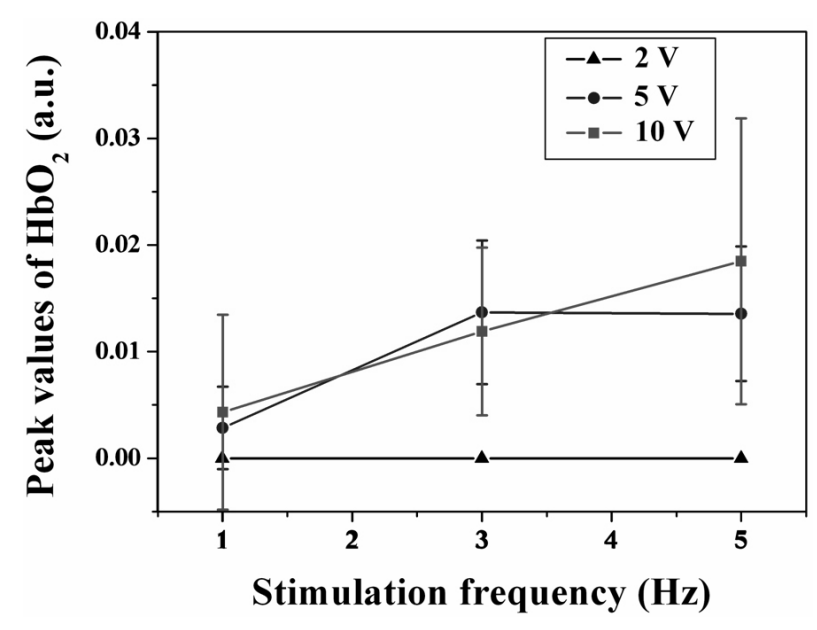

FIG. 6. Peak values of the $\mathrm{HbO}_{2}$, by various stimulation parameters

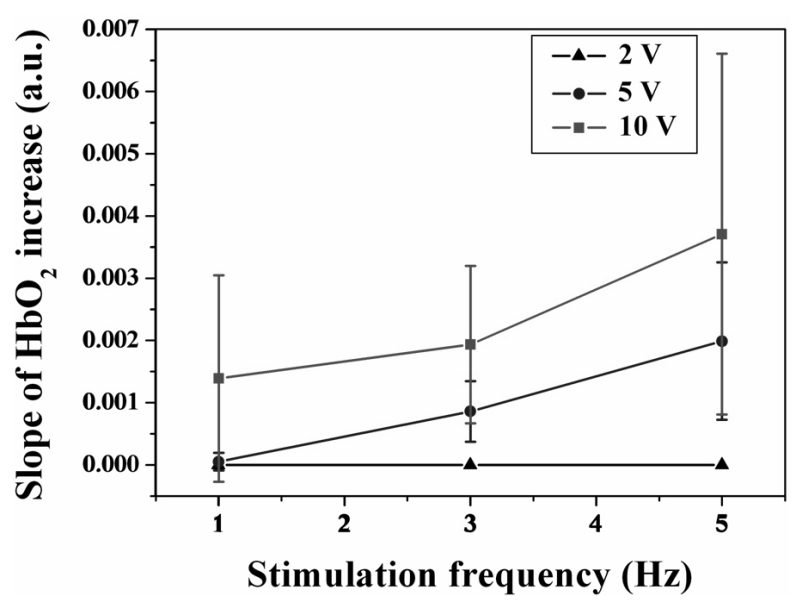

FIG. 7. Slope of the $\mathrm{HbO}_{2}$, by various stimulations

on any other areas but the left whisker.

As described earlier, three animals were used for three sets of consecutive experiments, each of which was separated by $3 \mathrm{~min}$ recovery time. The voltage levels and stimulation frequencies were 1,3 , and $5 \mathrm{~Hz}$ and 2,5 , and $10 \mathrm{~V}$, respectively. Therefore, the total number of data points was 81. Each set of $\mathrm{HbO}_{2}$ data was averaged and results are shown in Figs. 6 and 7.

As shown in Fig. 6, the amplitude of $\mathrm{HbO}_{2}$ change increases with stimulation frequencies. It seems that the amplitude change is less affected by the stimulation intensities. Also, it was observed that the cerebral hemodynamics changes were not changed when lower intensity stimulation $(2 \mathrm{~V})$ was applied regardless of stimulation frequencies. On the other hand, the $\mathrm{HbO}_{2}$ rises faster proportionally to the stimulation frequencies and intensities as shown in Fig. 7.

\section{DISCUSSION AND CONCLUSION}

Even if the diffuse optics has been used in human brain and breast studies for a long time, not many reports on small animal studies have been introduced. Our study shows that it is possible to measure hemodynamic responses in the barrel cortex of small animals using diffuse optical methods. Considering that the optical techniques are non-invasive and can be readily coupled with other imaging modalities, it will provide a unique tool for the neuroscience field.

Generally, after neurons are activated following appropriate stimulations, the blood volume that passes through the corresponding tissues increases. Then, due to metabolic demands, $\mathrm{HbO}_{2}$ concentration increases while the Hbr concentration decreases, which phenomenon is called a "wash-out effect" $[14,17]$. During the wash-out effect, the variations of $\mathrm{HbO}_{2}$ and $\mathrm{Hbr}$ concentrations are out of phase with each other [18]. Sometimes, when the supply of $\mathrm{HbO}_{2}$ is not sufficient to meet oxygen metabolism demands, there can be an initial dip in $\mathrm{HbO}_{2}$ concentration for a short time, followed by an increase (biphasic response) $[14,17]$. When neurons are activated, the increase in $\mathrm{HbO}_{2}$ is larger than the decrease in Hbr. As a result, the concentration of total hemoglobin $\left(\mathrm{HbO}_{2}+\mathrm{Hbr}\right)$ increases.

We observed similar trends in our experiments. As expected, the relationship between $\mathrm{HbO}_{2}$ and $\mathrm{Hbr}$ was out of phase, and the total hemoglobin concentration increased. However, we did not observe the aforementioned initial dip. Similar studies using a CCD camera that monitors the exposed brain cortex (skull is removed) and measures an optical intrinsic signal (OIS) have reported the biphasic phenomenon where $\mathrm{HbO}_{2}$ increases after an initial dip [19-20]. It may be because the barrel cortex is exposed directly to light during OIS measurement, which offers better resolution than non-invasive NIRS. Meanwhile, Blood et al. found via OIS study that when forepaw stimulation occurs at a higher frequency and for a longer period of time, the $\mathrm{HbO}_{2}$ concentration increases. The peak and slope values they reported are similar to our results [21].

Figs. 6 and 7 summarize our findings. It seems that the hemodynamic responses occur only when the stimulation exceeds certain threshold values. No NIRS signals were detected when $2 \mathrm{~V}$ electrical stimulation was applied. Electrical stimulations of $5 \mathrm{~V}$ and $10 \mathrm{~V}$ induced measurable hemodynamic responses. As shown in Fig. 7, the slope of $\mathrm{HbO}_{2}$ changes is proportional both to the stimulation frequencies and magnitude. On the other hand, the amplitude (Fig. 6) does not seem to change proportionally to the stimulation magnitude, while it responds proportionally to the stimulation magnitude. Three subjects may not be enough to make the final conclusion. In the future, we plan to increase the number of subjects with more stimulation parameters. In conclusion, the hemody- 
namic responses were successfully observed in the barrel cortex area using NIRS without the resection of the skull.

\section{ACKNOWLEDGMENTS}

This study was supported by a grant from the Korea Health 21 R\&D Project, Ministry of Health \& Welfare, Republic of Korea (A020607). This work was also supported by the Bio-signal Analysis Technology Innovation Program (M10645010001-06N4501-00110) of the Ministry of Science and Technology (MOST) and Korea Science and Engineering Foundation (KOSEF). This research was also supported by a grant (M103KV010023-08K220102310) from the Brain Research Center of the 21st Century Frontier Research Program, funded by the Ministry of Science and Technology, Republic of Korea.

\section{REFERENCES}

1. T. Misgeld and M. Kerschensteiner, "In vivo imaging of the diseased nervous system," Nat. Rev. Neurosci. 7, 44963 (2006).

2. J. Skoch, A. Dunn, B. T. Hyman, and B. J. Bacskai, "Development of an optical approach for noninvasive imaging of Alzheimer's disease pathology,” J. Biomed. Opt. 10, 11007 (2005).

3. J. A. Helpern, S. P. Lee, M. F. Falangola, V. V. Dyakin, A. Bogart, B. Ardekani, K. Duff, C. Branch, T. Wisniewski, M. J. de Leon, O. Wolf, J. O'Shea, and R. A. Nixon, "MRI assessment of neuropathology in a transgenic mouse model of Alzheimer's disease," Magn. Reson. Med. 51, 794-798 (2004).

4. F. F. Jobsis, "Noninvasive, infrared monitoring of cerebral and myocardial oxygen sufficiency and circulatory parameters," Science 198, 1264-1267 (1977).

5. A. Y. Bluestone, M. Stewart, J. Lasker, G. S. Abdoulaev, and A. H. Hielscher., "Three-dimensional optical tomographic brain imaging in small animals, part 1: hypercapnia,” J. Biomed. Opt. 9, 1046-1062 (2004).

6. J. P. Culver, T. Durduran, D. Furuya, C. Cheung, J. H. Greenberg, and A. G. Yodh, "Diffuse optical tomography of cerebral blood flow, oxygenation, and metabolism in rat during focal ischemia," J. Cereb. Blood Flow Metab. 23, 911-924 (2003).

7. A. M. Siegel, J. P. Culver, J. B. Mandeville, and D. A. Boas, "Temporal comparison of functional brain imaging with diffuse optical tomography and fMRI during rat forepaw stimulation," Phys. Med. Biol. 48, 1391-1403 (2003).

8. M. A. Franceschini, I. Nissila, W. Wu, S. G. Diamond, G. Bonmassar, and D. A. Boas, "Coupling between somat- osensory evoked potentials and hemodynamic response in the rat," Neuroimage 41, 189-203 (2008).

9. N. K. Logothetis, J. Pauls, M. Augath, T. Trinath, and A. Oeltermann, "Neurophysiological investigation of the basis of the fMRI signal," Nature 412, 150-157 (2001).

10. T. Matsuura and I. Kanno, "Quantitative and temporal relationship between local cerebral blood flow and neuronal activation induced by somatosensory stimulation in rats," Neurosci. Res. 40, 281-290 (2001).

11. A. Devor, A. K. Dunn, M. L. Andermann, I. Ulbert, D. A. Boas, and A. M. Dale, "Coupling of total hemoglobin concentration, oxygenation, and neural activity in rat somatosensory cortex," Neuron 39, 353-359 (2003).

12. M. Jones, N. Hewson-Stoate, J. Martindale, P. Redgrave, and J. Mayhew, "Nonlinear coupling of neural activity and CBF in rodent barrel cortex," Neuroimage 22, 956965 (2004).

13. A. Norup Nielsen and M. Lauritzen, "Coupling and uncoupling of activity-dependent increases of neuronal activity and blood flow in rat somatosensory cortex," J. Physiol. 533, 773-785 (2001).

14. D. G. Nair, “About being BOLD,” Brain Res. Rev. 50, 229-243 (2005).

15. C. I. Moore, S. B. Nelson, and M. Sur, "Dynamics of neuronal processing in rat somatosensory cortex," Trends. Neurosci. 22, 513-520 (1999).

16. D. T. Delpy, M. Cope, P. van der Zee, S. Arridge, S. Wray, and J. Wyatt, "Estimation of optical pathlength through tissue from direct time of flight measurement," Phys. Med. Biol. 33, 1433-1442 (1988).

17. M. Wolf, U. Wolf, V. Toronov, A. Michalos, L. A. Paunescu, J. H. Choi, and E. Gratton, "Different time evolution of oxyhemoglobin and deoxyhemoglobin concentration changes in the visual and motor cortices during functional stimulation: a near-infrared spectroscopy study, Neuroimage 16, 704-712 (2002).

18. S. Fantini, "A haemodynamic model for the physiological interpretation of in vivo measurements of the concentration and oxygen saturation of haemoglobin," Phys. Med. Biol. 47, N249-N257 (2002).

19. N. Prakash, J. D. Biag, S. A. Sheth, S. Mitsuyama, J. Theriot, C. Ramachandra, and A. W. Toga, "Temporal profiles and 2-dimensional oxy-, deoxy-, and total-hemoglobin somatosensory maps in rat versus mouse cortex," Neuroimage 37, S27-S36 (2007).

20. J. Mayhew, D. Johnston, J. Berwick, M. Jones, P. Coffey, and Y. Zheng, "Spectroscopic analysis of neural activity in brain: increased oxygen consumption following activation of barrel cortex," Neuroimage 12, 664-675 (2000).

21. A. J. Blood, S. M. Narayan, and A. W. Toga, "Stimulus parameters influence characteristics of optical intrinsic signal responses in somatosensory cortex, J J. Cereb. Blood Flow Metab. 15, 1109-1121 (1995). 